\title{
Fundamental origin of the large impact of strain on superconducting $\mathrm{Nb}_{3} \mathrm{Sn}$
}

\author{
A Godeke $^{1}{ }^{(1)}$, F Hellman $^{2,3}$, H H J ten Kate ${ }^{4,5}$ and M G T Mentink ${ }^{4}$ \\ ${ }^{1}$ Varian Medical Systems Particle Therapy GmbH, Troisdorf, Germany \\ ${ }^{2}$ University of California, Berkeley, CA, United States of America \\ ${ }^{3}$ Lawrence Berkeley National Laboratory, Berkeley, CA, United States of America \\ ${ }^{4}$ CERN, Geneva, Switzerland \\ ${ }^{5}$ University of Twente, Enschede, The Netherlands \\ E-mail: arno.godeke@varian.com
}

Received 27 March 2018, revised 3 August 2018

Accepted for publication 10 August 2018

Published 7 September 2018

\begin{abstract}
Superconductors can carry very high current densities without resistive loss. This makes them highly suitable for the construction of compact high field magnets of several tens of tesla. However, with increasing current density and magnetic field come high Lorentz loads and high strain levels, and it is empirically known that superconducting properties are affected by strain. The superconducting properties of the most commonly used high field material, $\mathrm{Nb}_{3} \mathrm{Sn}$, are particularly badly affected by strain. Here we demonstrate that strain causes significant sub-lattice distortion in the A15 lattice structure of $\mathrm{Nb}_{3} \mathrm{Sn}$, and show how this leads to the relatively large reduction of its superconducting properties. The changes are found to be primarily due to changes in the electron density of states, with a lesser contribution due to changes in the phonon spectrum. The amount of sub-lattice distortion further depends on crystal orientation. These findings suggest that it is possible to mitigate the reduction of the current carrying capacity by strain through crystal alignment and sub-lattice stabilization. This would enable superconducting magnets to reach a significantly higher magnetic field, and ease their construction by simplifying react-and-wind coil fabrication.
\end{abstract}

Keywords: $\mathrm{Nb}_{3} / \mathrm{Sn}, \mathrm{A} 15$ materials, strain, superconducting properties

(Some figures may appear in colour only in the online journal)

\section{Superconductors for high field magnets}

Science research and medical applications that use electromagnets, such as the high energy physics community; materials, physics, chemistry, and biology scientists using nuclear magnetic resonance; medical applications that use magnetic resonance imaging; and fusion programs such as the International Thermonuclear Experimental Reactor, all have a strong need for higher magnetic fields to enable a higher level of energy and enhanced resolution [1-3]. The use of superconductors poses major benefits for generating very high magnetic fields, due to their capacity to carry effective current densities that are three orders of magnitude higher than in normal-conducting materials since they do not generate heat due to ohmic loss.

The industry standard superconducting material is the ductile alloy of niobium and $46.5 \mathrm{wt} \%$ titanium, $\mathrm{Nb}-\mathrm{Ti}$ [4], which can transport current up to a critical current density, $J_{\mathrm{c}}$, of $3000 \mathrm{~A} \mathrm{~mm}^{-2}$ at $4.2 \mathrm{~K}$ and $5 \mathrm{~T}$. Its application is, however, limited by its upper critical magnetic field, $\mu_{0} H_{\mathrm{c} 2}$, of 14.5 T, and critical temperature, $T_{\mathrm{c}}$, of $9.2 \mathrm{~K}$. To generate magnetic fields beyond the limitation of $\mathrm{Nb}-\mathrm{Ti}$, one commonly switches to the intermetallic compound $\mathrm{Nb}_{3} \mathrm{Sn}[5,6]$ which, with a $\mu_{0} H_{\mathrm{c} 2}$ of $30 \mathrm{~T}$, a $T_{\mathrm{c}}$ of $18 \mathrm{~K}$, and a $J_{\mathrm{c}}$ of $3000 \mathrm{~A} \mathrm{~mm}^{-2}$ at $4.2 \mathrm{~K}$ and $12 \mathrm{~T}$ in the non-copper fraction, roughly doubles the available magnetic field and critical temperature. One can therefore generate more than twice the magnetic field in a solenoid with a given volume when using $\mathrm{Nb}_{3} \mathrm{Sn}$ instead of $\mathrm{Nb}-\mathrm{Ti}$, since the central magnetic field is to first order equal to the current density, $J$, multiplied by the windings thickness.

$\mathrm{Nb}_{3} \mathrm{Sn}$ is, similar to $\mathrm{Nb}-\mathrm{Ti}$, commonly made available as about $1 \mathrm{~mm}$ diameter wires in which a few to a few tens of $\mu \mathrm{m}$ diameter twisted filaments are embedded in a stabilizing, 


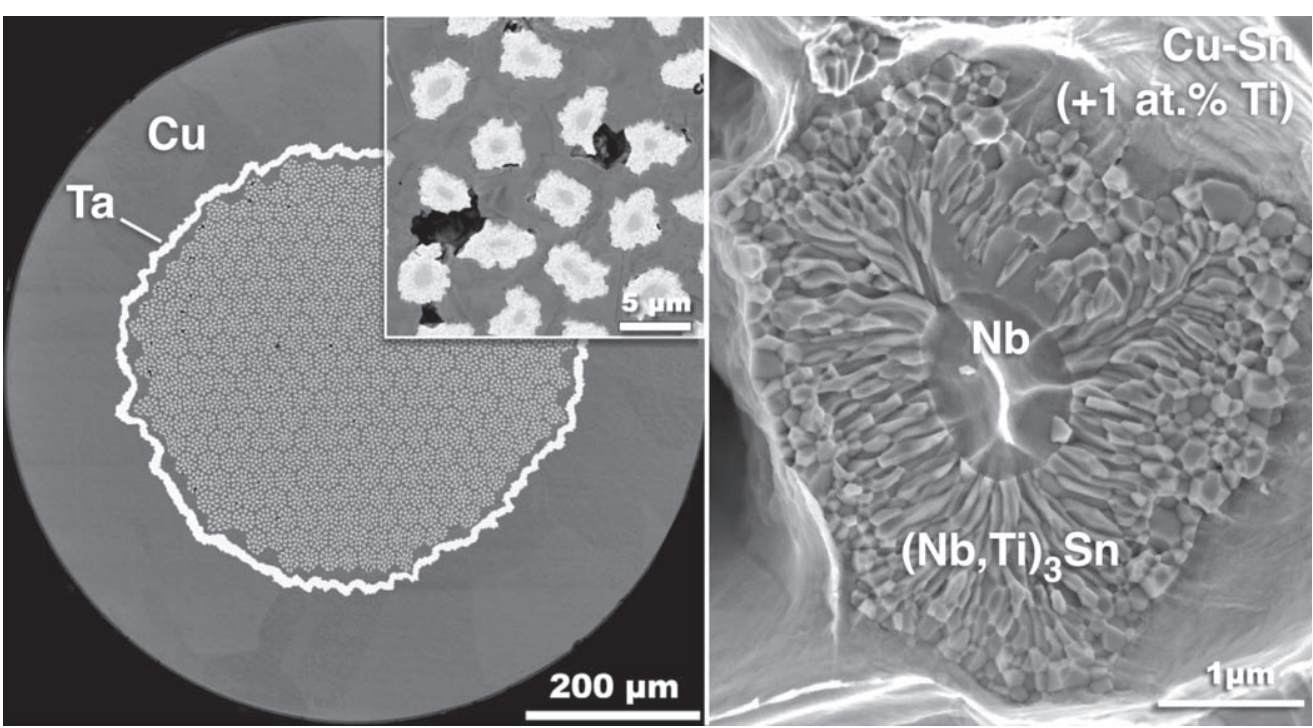

Figure 1. Backscatter electron micrograph from a scanning electron microscope ( $\mathrm{SEM}$ ) of a cross-section of a typical bronze-process $\mathrm{Nb}_{3} \mathrm{Sn}$ wire (left) manufactured by Furukawa Electric Co. Ltd after a diffusion heat treatment at $650{ }^{\circ} \mathrm{C}$ for $240 \mathrm{~h}$. Nb filaments are embedded in a $\mathrm{Cu}-\mathrm{Sn}$ bronze matrix to which $1 \mathrm{at} \% \mathrm{Ti}$ is added. The bronze is surrounded by a Ta diffusion barrier and embedded in a high purity $\mathrm{Cu}$ matrix. During the $\mathrm{Nb}_{3} \mathrm{Sn}$-forming heat treatment, $\mathrm{Sn}$ and Ti diffuse into the $\mathrm{Nb}$ filaments to form a ternary (Nb, Ti) ${ }_{3} \mathrm{Sn}$ layer (right) through a solid-state diffusion reaction. SEM cross-section taken by P J Lee and adapted from [7].

mostly copper, matrix (figure 1). In contrast to ductile $\mathrm{Nb}-\mathrm{Ti}$, $\mathrm{Nb}_{3} \mathrm{Sn}$ is brittle, and wires (and mostly also magnets) are fabricated while the $\mathrm{Nb}$ and $\mathrm{Sn}$ are still separated and ductile in the wire cross-section. The $\mathrm{Nb}_{3} \mathrm{Sn}$ is then formed after coil winding through a solid-state diffusion reaction at around $650{ }^{\circ} \mathrm{C}$ in an inert atmosphere of argon gas or vacuum. During this heat treatment, the Sn diffuses from a Sn source into the $\mathrm{Nb}$ and reacts to form the brittle $\mathrm{Nb}_{3} \mathrm{Sn}$ phase. A number of different wire processes exist. An example of a wire that is manufactured using the so-called bronze-process (in which a Sn-rich bronze is used as the Sn source) is shown in figure 1.

\section{Intermetallic niobium-tin}

Although mostly referred to $\mathrm{as} \mathrm{Nb}_{3} \mathrm{Sn}$ and forming in the $\mathrm{A} 15$ lattice structure, in practice it rarely occurs in the stoichiometric composition since the A15 phase is stable from about 18 to 25 at\% Sn [5, 6]. Due to this, the solid-state diffusion principally leads to $\mathrm{Sn}$ gradients, with the resulting A15 composition being Sn-rich close to the Sn source, but Sndepleted further away from the source [8]. Both $H_{\mathrm{c} 2}$ and $T_{\mathrm{c}}$ increase significantly with increasing $\mathrm{Sn}$ content and peak around 24.5 at $\% \mathrm{Sn}$ at $30 \mathrm{~T}$ and $18 \mathrm{~K}$, respectively [9]. The highest current density wires are therefore designed to achieve large volume-fractions of Sn-rich A15, so that the superconducting properties are retained in as much superconductor as possible when the magnetic field or temperature increases [10]. Above 24.5 at $\% \mathrm{Sn}$ a lattice instability triggers a spontaneous cubic-to-tetragonal transition, which causes a collapse of $H_{\mathrm{c} 2}$ [11]. For this reason $\mathrm{Nb}_{3} \mathrm{Sn}$ is often alloyed with a ternary element (mostly $\mathrm{Ti}$ or $\mathrm{Ta}$ ), which cause an increase of the electrical resistivity and a stabilization of the high- $H_{\mathrm{c} 2}$ cubic phase. At present, $\mathrm{Nb}_{3} \mathrm{Sn}$ seems fully optimized in terms of composition, which determines its $H_{\mathrm{c} 2}$ and $T_{\mathrm{c}}[5,6,11]$.

$\mathrm{Nb}_{3} \mathrm{Sn}$ is, like $\mathrm{Nb}-\mathrm{Ti}$, a so-called Type 2 superconductor, in which magnetic flux quanta in the shape of flux lines penetrate its interior when the external magnetic field is higher than the lower critical magnetic field $H_{\mathrm{c} 1}$. The flux-line density increases with the external magnetic field until the flux lines start to overlap, thereby defining $H_{\mathrm{c} 2}$. Carrying a transport current causes a gradient in the flux-line density, and a force, which is perpendicular to the current, on the flux lines to re-establish a homogeneous distribution. Following Maxwell's equations, flux movement perpendicular to the current will cause an electric field parallel to the current and thus ohmic loss. This has to be prevented by pinning the flux lines at grain boundaries or defects. The pinning force that can be provided determines the current density $J_{\mathrm{c}} . \mathrm{Nb}_{3} \mathrm{Sn}$ is, in contrast to $\mathrm{Nb}-\mathrm{Ti}$, not yet fully optimized in terms of flux pinning due to the absence of sufficient pinning centers, and gains of a factor of two to three in the current density $J_{\mathrm{c}}$ at $12 \mathrm{~T}$ and $4.2 \mathrm{~K}$ are within reach [12].

A complication when carrying a high current density in a high magnetic field is the high Lorentz force, $\overrightarrow{\mathbf{F}_{\mathrm{L}}} \equiv \overrightarrow{\mathbf{J}} \times \overrightarrow{\mathbf{B}}$, that acts on the superconducting wires. This load causes, through the compliance of the conductor and supporting structure, a significant level of deformation, or strain, $\epsilon$, on the superconducting material that comes in addition to manufacturing and thermal strain. From experiments it is well known that the superconducting properties are, to a greater or lesser extent, affected by strain. Measurements of the strain sensitivity are most commonly done by imposing a uniaxial strain on the superconductor, characteristically in the range of 


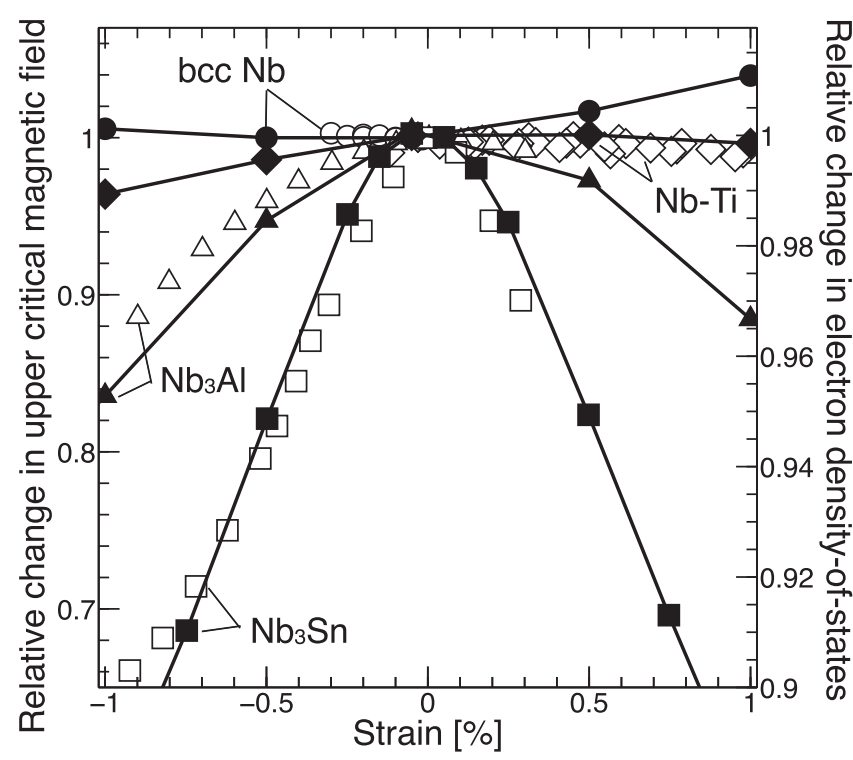

Figure 2. Experimentally observed variations of the upper critical magnetic field with strain of bcc $\mathrm{Nb}$ (this work), $\mathrm{Nb}-\mathrm{Ti}$ [15], and $\mathrm{Nb}_{3} \mathrm{Al}$ and $\mathrm{Nb}_{3} \mathrm{Sn}$ [14] (open symbols), compared to calculated changes in the electron density of states (DOS) (closed symbols).

$-1 \%$ to $+0.5 \%$. This strain range covers the strain levels that are generated in magnets under operating conditions. For $\mathrm{Nb}_{3} \mathrm{Sn}$ it is found that, despite the material's brittleness, a reversible change in the superconducting properties occurs until the $\mathrm{Nb}_{3} \mathrm{Sn}$ fractures, which usually takes place in tension. A number of empirical descriptions exist in the literature to describe the behavior of the critical current density as a function of strain $J_{\mathrm{c}}(\epsilon)$ for $\mathrm{Nb}_{3} \mathrm{Sn}$ (see for example [13] and the references therein), but until now a comprehensive explanation of the principle physical origin of the changes in the superconducting properties with strain has not been published.

The typical loss of $J_{\mathrm{c}}$ at $0.5 \%$ uniaxial compressive or tensile strain at $4.2 \mathrm{~K}$ for $\mathrm{Nb}_{3} \mathrm{Sn}$ is about $50 \%$ for a magnetic field around $\frac{1}{2} H_{\mathrm{c} 2}$. This loss in critical current density $J_{\mathrm{c}}$ results in more bulky coil windings unless the strain can be limited, and it stems from a reduction in the upper critical magnetic field $H_{\mathrm{c} 2}(T)$, which in turn limits the achievable magnetic field. Notably, the reduction in the superconducting properties of $\mathrm{Nb}_{3} \mathrm{Sn}$ is about twice that of $\mathrm{Nb}_{3} \mathrm{Al}$ [14], which is a comparable superconductor that also has the A15 crystal structure, but is metastable and, hence, much more difficult to produce. The strain sensitivity of $\mathrm{Nb}_{3} \mathrm{Sn}$ is in addition more than one order of magnitude higher than in other Nb-based superconductors such as $\mathrm{Nb}-\mathrm{Ti}$ and $\mathrm{Nb}$ (shown in figure 2). A fundamental understanding of why these differences in the sensitivity to strain are so large is critical to finding methods to reduce the strain sensitivity of $\mathrm{Nb}_{3} \mathrm{Sn}$. We therefore performed a comprehensive analysis of the origin of the strain dependence of $\mathrm{Nb}_{3} \mathrm{Sn}$ using $a b$ initio and microscopic calculation without a priori assumptions on the physical origin of the strain sensitivity (in contrast to earlier attempts by others). We compare results to $\mathrm{Nb}$ and $\mathrm{Nb}_{3} \mathrm{Al}$ and validate our calculations with new measurements and experimental results from the literature.

\section{Methods}

\subsection{Ab initio and microscopic calculations}

The materials shown in figure 2 are all phonon-mediated superconductors. This means that free electrons, whose availability is captured in the electron DOS at the Fermi energy level, $N\left(E_{\mathrm{F}}\right)$, form superconducting Cooper pairs [16] by coupling through the vibrating ions in the lattice. The magnitude by which these ions can vibrate at various frequencies is described by the phonon spectrum, or phonon DOS as a function of frequency, $F(\omega)$. The detailed interaction between the electrons and the vibrating ions, again as a function of frequency, is described by the electron-phonon coupling characteristic, $\alpha^{2}(\omega)$, which includes the density of electrons and the ion mass. The product $\alpha^{2}(\omega) F(\omega)$ is referred to as the Éliashberg function [17] and captures the electronic and vibrational properties of the material. From this function, an electron-phonon coupling constant, $\lambda$, can be calculated through:

$$
\begin{aligned}
\lambda=2 \int \frac{\alpha^{2}(\omega) F(\omega)}{\omega} d \omega & =\frac{N\left(E_{\mathrm{F}}\right)\left\langle I^{2}\right\rangle}{\left\langle\omega^{2}\right\rangle M}, \\
\text { with } \int F(\omega) d \omega & \equiv 1 .
\end{aligned}
$$

The right side of equation (1) was proposed by McMillan [18] and Hopfield [19] in which $\left\langle\omega^{2}\right\rangle$ is the average of the squared vibrational frequency:

$$
\left\langle\omega^{2}\right\rangle \equiv \frac{2}{\lambda} \int \alpha^{2}(\omega) F(\omega) \omega d \omega,
$$

$\left\langle I^{2}\right\rangle$ is the mean electronic matrix element, and $M$ is the effective ion mass. After a correction for the Coulomb repulsion between electrons, $\mu^{*}$, leading to an effective electron-phonon coupling constant, $\lambda_{\text {eff }}$, the critical temperature can be calculated through [20]:

$$
T_{\mathrm{c}}=\frac{0.25\left\langle\omega^{2}\right\rangle^{0.5}}{\sqrt{e^{2 / \lambda_{\mathrm{eff}}}-1}},
$$

in which $e$ is the elementary charge.

The upper critical magnetic field can be calculated using the microscopic theory for phonon-mediated superconductivity as developed by Ginzburg and Landau [21], Abrikosov [22], and Gor'kov [23]. The normal-state resistivity, $\rho_{\mathrm{n}}$, just above the critical temperature $T_{\mathrm{c}}$ of stoichiometric $\mathrm{Nb}_{3} \mathrm{Sn}$ is below $5 \mu \Omega \mathrm{cm}$ [5]. This is generally referred to as a clean limit, for which the mean scattering time between electron collisions, $\tau$, is large, leading to a long mean-freepath, $l_{\mathrm{mfp}}$, for a given velocity of the electrons around the Fermi energy, $\nu_{\mathrm{F}}=l_{\mathrm{mfp}} / \tau$. For the mainly off-stoichiometric A15 phase that occurs in wires, $\rho_{\mathrm{n}}$ gradually increases with reducing Sn content to around $90 \mu \Omega \mathrm{cm}$ at 18 at $\%$ Sn [5], which is referred to as dirty with a short mean-free-path. Calculations of $H_{\mathrm{c} 2}$ have therefore been performed for the 
intermediate to dirty range, with corrections for strong coupling between the electrons and for Pauli paramagnetic limiting of $H_{\mathrm{c} 2}$. We previously demonstrated that calculating $H_{\mathrm{c} 2}$ in this way accurately agrees with experimental results on a wide range of samples of various compositions and morphologies [11]. Additional corrections [24] were found insignificant, but for a discussion of these and the details of the calculations of the upper critical magnetic field $H_{\mathrm{c} 2}$ we refer to our earlier publication [11].

The electronic properties and the phonon spectrum, as well as their variation with strain, were calculated using density functional theory (DFT) and density functional perturbation theory in QUANTUM ESPRESSO [25], using a stoichiometric $\mathrm{Nb}_{3} \mathrm{Sn}$ unit cell of six $\mathrm{Nb}$ and two $\mathrm{Sn}$ atoms in an A15 crystal structure. For the details of these calculations we again refer to our earlier publication in which we describe results for disordered $\mathrm{Nb}_{3} \mathrm{Sn}$ [11]. The calculations require a huge amount of processing power and were therefore performed using the computing resources at the Cornell NanoScale Science and Technology Facility and Berkeley's National Energy Research Scientific Computing Center. A large number of calculations at various strain states were needed. A complication arised since calculations for offstoichiometric $\mathrm{Nb}_{3} \mathrm{Sn}$ require calculations on large super cells to accommodate the 18-25 at\% Sn composition range, which would have required excessively large computational time. To retain manageable calculations, three approximations were used.

For our first approximation, we note that off-stoichiometry creates disorder in the lattice, increased scattering, a shortening of the mean-free-path length $l_{\mathrm{mfp}}$, and an increase of the normal-state resistivity $\rho_{\mathrm{n}}$. Following others [26, 27] and our earlier work [11], we simulated such disorder by introducing electron lifetime broadening. This creates an uncertainty in the energy of the electrons to simulate the increased amount of scattering, which can mathematically be described by [27]:

$$
E_{\mathrm{b}}=\frac{\hbar}{\tau}
$$

in which $E_{\mathrm{b}}$ is the energy broadening and $\hbar$ is the reduced Planck constant. We demonstrated previously by comparison to experimental results and super-cell calculations that this approach to simulate disorder while retaining unit-cell calculations yields correct values for the critical temperature $T_{\mathrm{c}}$ and the upper critical magnetic field $H_{\mathrm{c} 2}$ as a function of composition, and is a reasonable approach to describe disorder in general [11]. We will therefore approximate the effects of off-stoichiometric composition to be dominated by the disorder it produces.

Our second approximation concerns the derivation of a function to describe the electron-phonon coupling characteristic for a multiple ion system, which we will refer to as $\alpha_{\text {eff }}^{2}(\omega)$. By combining (1)-(3) it is found that for single ion superconductors:

$$
\alpha^{2}(\omega)=\alpha_{\mathrm{IM}}^{2} \frac{N\left(E_{\mathrm{F}}\right)}{\omega}
$$

with $\alpha_{\mathrm{IM}}^{2}=\left\langle I^{2}\right\rangle / 2 M$, a constant. For a multiple ion system, however, it is non-trivial to determine how the electrons couple to the individual ion species. As a solution, one can assume a weighted summation of the individual ion contributions [11] and combine that with experimental observations [28]. In this way, it is found for $\mathrm{Nb}_{3} \mathrm{Sn}$ that $\alpha_{\text {eff }}^{2}(\omega) \propto \exp \left(-\omega / \omega_{0}\right)$, and one can derive an empirical expression for the electron-phonon interaction characteristic of $\mathrm{Nb}_{3} \mathrm{Sn}$ :

$$
\alpha_{\mathrm{eff}}^{2}(\omega)=\alpha_{\mathrm{IM}, \mathrm{eff}}^{2} N\left(E_{\mathrm{F}}\right) \exp \left(-\omega / \omega_{0}\right)
$$

This relation has two global constants: a characteristic frequency $\omega_{0}$ of $14.2 \mathrm{meV}$, and a constant for a multiple ion system $\alpha_{\text {IM,eff }}^{2}$ of $2.08 \times 10^{-3} \mathrm{eV}^{2} /($ states $\times$ unit cell). The details of this derivation, as well as verifications of the validity of this approach were published previously [11].

Our third approximation is the assumption of a constant Coulomb repulsion $\mu_{0}^{*}$. Results from tunnel experiments show that $\mu_{0}^{*}$ is $0.13 \pm 0.02$ over the entire resistivity (and composition) range $[11,29]$, and the average $\mu_{0}^{*}$ calculated from five measured Éliashberg spectra of stoichiometric $\mathrm{Nb}_{3} \mathrm{Sn}$ is $0.125 \pm 0.04$ [11], which we will use as a global constant.

$A b$ initio calculations were performed using these approximations, starting from a strain-free, cubic A15 lattice. The DFT-determined minimum-energy cubic lattice constant is $0.5313 \mathrm{~nm}$, which is close to the value of $0.5290 \mathrm{~nm}$ that is measured in stoichiometric bulk $\mathrm{Nb}_{3} \mathrm{Sn}$ [30], and within the accuracy that can be expected from DFT calculations. The spontaneous tetragonal distortion occurs only above a critical mean scattering time $\tau_{\mathrm{c}}$ of $(1.53 \pm 0.08) \times 10^{-14} \mathrm{~s}$ with a root-mean-square velocity at the Fermi energy level $\nu_{\mathrm{F}, \mathrm{rms}}=(2.121 \pm 0.014) \times 10^{5} \mathrm{~m} \mathrm{~s}^{-1}$ and thus for $l_{\mathrm{mfp}}>$ $3.25 \pm 0.19 \mathrm{~nm}$ and $\rho_{\mathrm{n}}<\rho_{\mathrm{c}}=27.0 \pm 1.2 \mu \Omega \mathrm{cm} \mathrm{[11]}$.

The calculations were only performed for shorter meanfree-paths, since unit-cell calculations in the preferentially tetragonal regime give incorrect results [11] due to the occurrence of $c$-axis misalignments that cause so-called tweed patterns and local strain variations on a scale of $10 \mathrm{~nm}$. The effects of such local strain variations on the electronic properties are unclear, and investigating these requires calculations using very large super cells, which is computationally unrealistic.

We focus here on calculations for an $l_{\mathrm{mfp}}$ of $2.85 \mathrm{~nm}$, since for this mean-free-path length the critical temperature $T_{\mathrm{c}}$ is $16.7 \mathrm{~K}$ and the upper critical magnetic field $\mu_{0} H_{\mathrm{c} 2}$ is $28.1 \mathrm{~T}$ [11], which are values that are representative for wires $[31,32]$. Calculations of the effect of strain were performed also for other mean-free-paths and these show that the sensitivity to strain increases for cleaner material with a longer mean-free-path. Strain is applied in either the [100] or [110] direction to enable comparison to experimental results (below). A structural optimization, in which the out-of-plane lattice vector is free to expand or contract and all ions are allowed to assume the most energetically favorable position, is performed for every strain state. 
In addition to extensive calculations on $\mathrm{A} 15 \mathrm{Nb}_{3} \mathrm{Sn}$, the electronic DOS as a function of strain was also calculated for $\mathrm{A} 15 \mathrm{Nb}_{3} \mathrm{Al}$ and for bcc $\mathrm{Nb}$ and $\mathrm{Nb}-\mathrm{Ti}$ to enable comparisons to their sensitivity to strain. Calculations of structural optimizations with strain in the [100] direction were performed on all these materials, after which $N\left(E_{\mathrm{F}}\right)$ was calculated. The mean-free-path for $\mathrm{Nb}_{3} \mathrm{Al}$ was fixed to $l_{\mathrm{mfp}}=2.85 \mathrm{~nm}$. For $\mathrm{Nb}$ and $\mathrm{Nb}-\mathrm{Ti}$ an $l_{\mathrm{mfp}}$ of $6.5 \mathrm{~nm}$ was used, corresponding to the experimentally observed $\rho_{\mathrm{n}}$ of $5.6 \mu \Omega \mathrm{cm}$ for the $\mathrm{Nb}$ sample in figure 2.

\subsection{Experiments}

To validate our calculations, we compared to experimental data from literature, and to own measurements on bulk material, thin films, and wires. Details of sample preparation and initial characterization of the bulk and thin film samples, including our finding that the normal-state resistivity $\rho_{\mathrm{n}}$ is significantly affected by strain [33], were published earlier [33-35], with further experimental characterization to be published elsewhere [36]. Here we summarize the sample details that are relevant for our present analyses.

Thin film and bulk samples were mounted by glueing them on a Ti-6Al-4V substrate to which strain can be applied and on which voltages as a function of transport current can be measured at various magnetic fields and temperatures. It has been shown before that the macroscopic strain is correctly transferred to the $\mathrm{Nb}_{3} \mathrm{Sn}$ crystals in such experiments [37]. A polyimide film is placed in between a sample and the holder, so that the normal-state resistivity of the superconductor remains accessible, which is important for correlations to the microscopic theory.

The transfer of the longitudinal in-plane applied strain was confirmed using strain-gauges mounted on top of the sample. For the strain in the transverse in-plane direction of the thin films, which is mainly determined by the Poisson ratio of 0.342 of the Ti-6Al-4V substrate material, a Poisson ratio of $-0.340 \pm 0.007$ was measured. Strain in the free outof-plane transverse direction is determined by the Poisson ratio of the $\mathrm{Nb}_{3} \mathrm{Sn}$, which is around $0.45[37,38]$. The effective Poisson ratio for the radial direction in $\mathrm{Nb}_{3} \mathrm{Sn}$ inside wires, in which its magnitude is reduced due to the presence of the matrix materials, is between 0.35 and 0.38 , depending on wire type $[39,40]$. It can therefore be concluded that, at least to first order, the three-dimensional strain state in model samples, wires, and calculations, will be similar when a onedimensional deformation is induced.

The crystal orientation in the thin films, as measured by x-ray diffraction, is preferentially [001] in the out-of-plane direction and the in-plane orientation is random. This means that the $\mathrm{Nb}_{3} \mathrm{Sn}$ grains in the film are exposed to either a [100] or a [110] strain, or a direction in between these limits. Crystal orientation analyses on wires indicate a [100] or [110] preferential orientation in the $\mathrm{Nb}_{3} \mathrm{Sn}$ parallel to the wire axis, presumably at least partially as a result of the strong texture that is present in the $\mathrm{Nb}$ and $\mathrm{Nb}-\mathrm{Ta}$ precursors due to wire drawing [41]. Since the strain in the experimental results is predominantly in the [100] or [110] direction or any direction

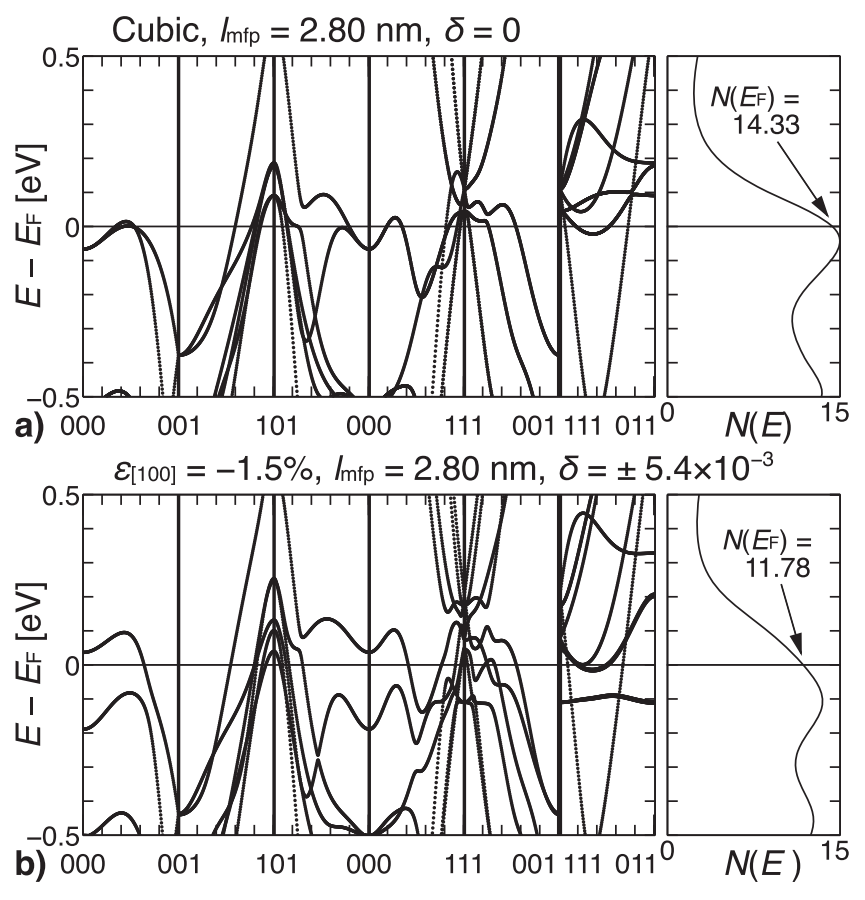

Figure 3. Calculated electronic band structure (left) and DOS as a function of energy (right) for a cubic $\mathrm{Nb}_{3} \mathrm{Sn}$ lattice (a) and for a lattice that is deformed to $-1.5 \%$ strain in the [100] direction, resulting in a sub-lattice distortion $\delta= \pm 5.4 \times 10^{-3}(\mathrm{~b})$.

in between, we limit our calculations to strain applied in these directions.

\section{Findings}

In figure 3 the band structure and the electron DOS are shown for the entire Brillouin zone under zero strain, and when the crystal is subjected to $-1.5 \%$ strain along the [100] direction. Substantial differences are seen, including most notably a decrease in $N\left(E_{\mathrm{F}}\right)$ from 14.33 to 11.78 states $(\mathrm{eV} \times \mathrm{uc})^{-1}$. Note that the band structures are calculated for a mean-freepath of $2.80 \mathrm{~nm}$, whereas in our other figures an $l_{\mathrm{mfp}}$ of $2.85 \mathrm{~nm}$ is used. A larger $l_{\mathrm{mfp}}$ results in slightly increased values for $N\left(E_{\mathrm{F}}\right)$. A summary of $N\left(E_{\mathrm{F}}\right)$ as a function of $\epsilon_{[100]}$ over the practically relevant strain range is shown in figure 4.

A remarkable effect is observed in this [100] strain state: the $\mathrm{Nb}$ ions in the directions perpendicular to the strain change their relative position inside the lattice, moving towards or away from each other as strain is applied, while the relative position of the $\mathrm{Nb}$ ions along the strain direction are largely unaffected, i.e. the two $\mathrm{Nb}$ chains perpendicular to the strain direction ([010] and [001]) dimerize, while the ones along the [100] strain direction remain equally spaced (there is an overall tetrahedal distortion of the originally cubic cell, so the $\mathrm{Nb}$ spacings are increase, but they remain equally spaced). This effect, which emerges from the minimization of energy in the computed structural optimizations and which we will refer to as sub-lattice distortion, is sketched in the inset of figure 4 . The phenomenon is notably the same as the rearrangement of the $\mathrm{Nb}$ ions during the spontaneous 


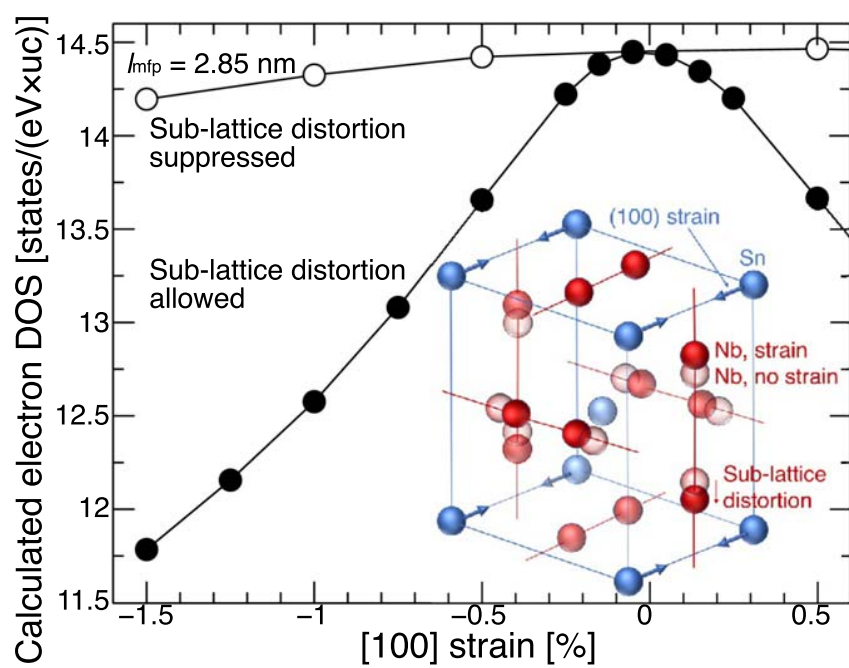

Figure 4. Calculated changes in the electron DOS with and without suppression of the sub-lattice distortion. In the inset, the $\mathrm{A} 15 \mathrm{Nb}_{3} \mathrm{Sn}$ lattice to which strain in the [100] direction is applied is schematically shown. The resulting sub-lattice distortion in the $\mathrm{Nb}$ chains is also indicated (e.g. $\mathrm{Nb}$, strain) with the positions without sub-lattice distortion depicted as a lighter shaded color (e.g. Nb, no strain).

cubic-to-tetragonal distortion that occurs for low resistivity, stoichiometric samples [11, 42-44].

The sub-lattice distortion, $\delta$, expressed as a fraction of the lattice parameter, is $-5.4 \times 10^{-3}$ and $+5.4 \times 10^{-3}$ at a strain $\epsilon_{[100]}$ of $-1.5 \%$ for each of the two transverse directions for an $l_{\mathrm{mfp}}$ of $2.85 \mathrm{~nm}$. For $\mathrm{Nb}_{3} \mathrm{Sn}$ with about half the meanfree-path, for an $l_{\mathrm{mfp}}$ of $1.3 \mathrm{~nm}$, the sub-lattice distortion also approximately halves to $\pm 3 \times 10^{-3}$ for the same strain level. Thus, the sub-lattice distortion due to strain is larger for cleaner $\mathrm{Nb}_{3} \mathrm{Sn}$ with a higher $T_{\mathrm{c}}$ and $H_{\mathrm{c} 2}$. An important effect occurs when the sub-lattice distortion is artificially suppressed: the change in $N\left(E_{\mathrm{F}}\right)$ for any given strain is reduced by more than one order of magnitude. This means that the $\mathrm{Nb}$ sub-lattice distortion is the leading cause (for about 90\%) for the reduced $N\left(E_{\mathrm{F}}\right)$. Similar effects are seen for [110] strain and for other directions, although the sub-lattice distortions are more complex [36].

Next to a change in the electron DOS, also the phonon spectrum is affected by strain, as is shown in the inset of figure 5 for zero strain and $-1.5 \%$ strain in the [100] direction: compared to the spectrum for the strain-free crystal, the phonon DOS peaks are somewhat reduced in amplitude and shifted to higher frequencies when the crystal is under $-1.5 \%$ strain in the [100] direction. To investigate the relative contributions of the change in the electronic properties and the phonon spectrum with strain, $H_{\mathrm{c} 2}(\epsilon, T=0 \mathrm{~K})$ was calculated by allowing only the electronic properties to change while fixing $F(\omega)$ at the strain-free value, and comparing that to a calculation of $H_{\mathrm{c} 2}(\epsilon)$ in which both the phonon and electron DOS are allowed to change with strain. The result of this comparison is shown in figure 5 , and it is seen that for strain in the [100] direction, the change in $H_{\mathrm{c} 2}$ is $85 \%$ attributable to the change in the electronic properties, and only $15 \%$ is attributable to changes in the phonon spectrum. For strain in

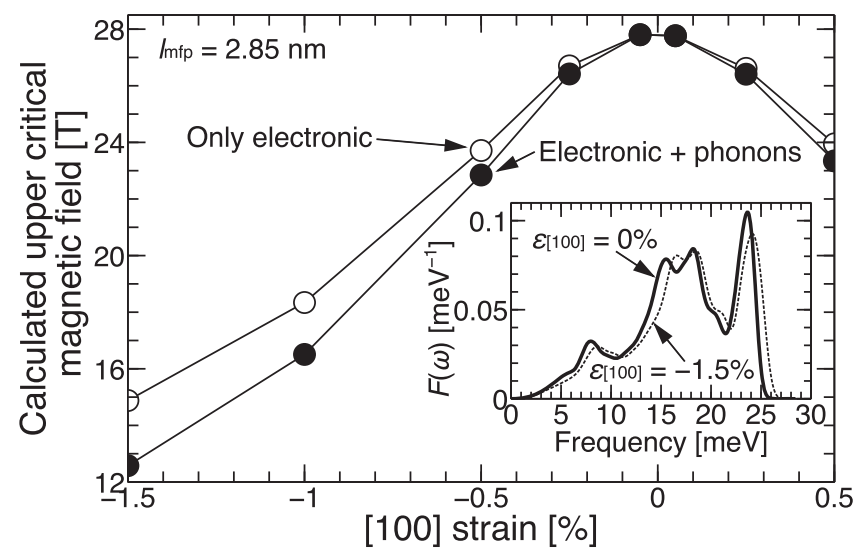

Figure 5. Calculated changes in the upper critical magnetic field with strain due to changes in the electron DOS alone, and due to changes in the electron DOS and phonon spectrum combined. The inset shows the change in the phonon spectrum between $\epsilon_{[100]}=0 \%$ and $-1.5 \%$.

the [110] direction, the change in $H_{\mathrm{c} 2}$ is for $77 \%$ attributable to a change in the electronic properties. These findings are in contrast to other analyses in which mostly an a priori assumption was made that changes in the phonon spectrum [28, 45-49], or changes in the electron DOS [50-52], are the dominant cause for the large strain sensitivity of $\mathrm{Nb}_{3} \mathrm{Sn}$. We also note that an assumption that changes in the phonon spectrum dominate the effects of strain, is in conflict with the experimental evidence that $\rho_{\mathrm{n}}$ changes substantially with strain $[33,36]: \rho_{\mathrm{n}}$ is at temperatures below $18 \mathrm{~K}$ mainly determined by defect scattering and not by the phonons.

We now consider the effect of strain on $N\left(E_{\mathrm{F}}\right)$ for three other phonon-mediated superconductors: bcc $\mathrm{Nb}$ and $\mathrm{Nb}-\mathrm{Ti}$, and specifically for $\mathrm{A} 15 \mathrm{Nb}_{3} \mathrm{Al}$, with its similar crystal structure but approximately half the sensitivity to strain compared to $\mathrm{Nb}_{3} \mathrm{Sn}$ [14]. The resulting $N\left(E_{\mathrm{F}}, \epsilon\right)$ are compared with experimental results for $H_{\mathrm{c} 2}(\epsilon)$ in figure 2. Despite only using $N\left(E_{\mathrm{F}}\right)$ as a proxy for $H_{\mathrm{c} 2}$, a striking agreement is found between the relative changes in experimental data for $H_{\mathrm{c} 2}(\epsilon)$ and the calculated $N\left(E_{\mathrm{F}}, \epsilon\right)$.

The calculated $N\left(E_{\mathrm{F}}, \epsilon\right)$ of $\mathrm{Nb}_{3} \mathrm{Al}$ also shows, similar to what was experimentally found for $H_{\mathrm{c} 2}(\epsilon)$, approximately half the sensitivity to strain compared to the $N\left(E_{\mathrm{F}}, \epsilon\right)$ of $\mathrm{Nb}_{3} \mathrm{Sn}$. This difference is at least in part due to the lower degree of sub-lattice distortion in $\mathrm{Nb}_{3} \mathrm{Al}$ which, at $-1 \%$ compressive strain, amounts to $\pm 3.6 \times 10^{-3}$ compared to $\pm 4.3 \times 10^{-3}$ for $\mathrm{Nb}_{3} \mathrm{Sn}$. The resulting electron DOS for $\mathrm{Nb}_{3} \mathrm{Al}$ with a mean-free-path of $2.85 \mathrm{~nm}$ changes from 12.88 to 12.24 states $(\mathrm{eV} \times \mathrm{uc})^{-1}$ when applying $-1 \%$ [100] strain. $\mathrm{Nb}$ and $\mathrm{Nb}-\mathrm{Ti}$ do not have the characteristic $\mathrm{Nb}$ chains that are present in the A15 lattices of $\mathrm{Nb}_{3} \mathrm{Sn}$ and $\mathrm{Nb}_{3} \mathrm{Al}$ and their sensitivity to strain is lower.

As a further verification of our calculation results for $\mathrm{Nb}_{3} \mathrm{Sn}$, we make a comparison to experimental literature data of $H_{\mathrm{c} 2}(\epsilon)$ for seven different wires, as shown in figure 6. A comparison to experimental results is shown for the calculated $H_{\mathrm{c} 2}(\epsilon)$ with strain in the [100] and the [110] directions, as well as their average. It is found that this average is close to the experimental values, suggesting that the average crystal 


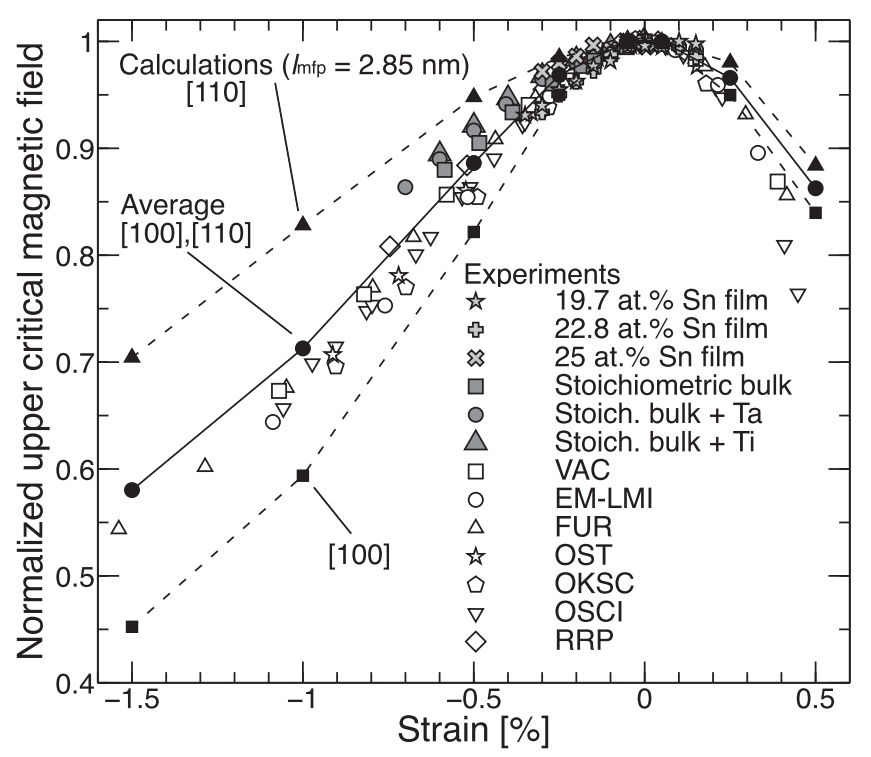

Figure 6. Calculated sensitivity of the upper critical magnetic field to strain applied in the [100] and [110] crystal directions and the averaged [100] and [110] sensitivities (closed symbols), compared to experimental results of the upper critical magnetic field as a function of longitudinal applied strain on practical conductors manufactured through various processes [31, 53-55] (open symbols), as well as selected data on thin film and bulk samples from our present and previous work $[34,36]$ (gray symbols).

orientation in wires along the wire axis is roughly halfway between [100] and [110] with a slight tendency towards the [100] direction.

Measurements of the crystal orientation in powder-intube (PIT) and Restack Rod Processed (RRP ${ }^{\circledR}$ ) wires indicate that there is [110] texture along the wire axis in PIT wires (a factor 10 above random), whereas there is a [100] texture (a factor of 14 above random) along the wire axis in $\mathrm{RRP}^{\circledR}$ wires [41]. For bronze-processed wires that are similar to the wire depicted in figure 1, a slight [110] texture (a factor of 1.7 above random) has been reported [56]. These findings would suggest a reduced strain sensitivity for PIT processed wires compared to $\mathrm{RRP}^{\circledR}$ wires, with bronze-processed wires somewhere in between, but in the limited $H_{\mathrm{c} 2}(\epsilon)$ dataset shown in figure 6 this is not visible. The reason for this apparent discrepancy is, for now, unclear, and further dedicated strain experiments that focus on the relative sensitivities of different crystal orientations will be needed to support or disprove our findings.

More extensive analyses on the strain sensitivity of thin films and bulk materials will be published in the near future, but for completeness we already introduce a selection of our samples in figure 6. For the thin film results, we selected films with $19.7,22.8$, and 25 at $\% \mathrm{Sn}$, which have $\mu_{0} H_{\mathrm{c} 2}$ values of 19.1, 27.9, and $30.4 \mathrm{~T}$, respectively. We also selected stoichiometric binary bulk, and stoichiometric binary bulk with 4 at $\% \mathrm{Ta}$ or $1.5 \mathrm{at} \% \mathrm{Ti}$, with $\mu_{0} H_{\mathrm{c} 2}$ values of $27.0,29.3$, and $30.0 \mathrm{~T}$, respectively.

Although the experimental strain range is limited, two trends can be distinguished: the off-stoichiometric thin films have a similar strain sensitivity to the wires, which is in-line with their random [110]-[100] in-plane crystal orientation, and the off-stoichiometric A15 compositions that also determine the critical properties in wires. For the stoichiometric thin film and bulk samples a reduced strain sensitivity is observed, which is in-line with our observations that when approaching stoichiometry, the sensitivity to strain rapidly reduces (see figure 2.24 in [36]). The reason for this reduced sensitivity, however, should not be sought in crystal alignment, but in the presence of the spontaneous tetragonal distortion, which affects the way that externally applied strain enters the lattice structure. An exact and detailed explanation of how this results in a reduced sensitivity can, for now, only be hypothesized, due to our inability to perform reliable calculations when the spontaneous tetragonal distortion occurs, as explained above. An accurate analysis is moreover complicated by the sample inhomogeneities that inevitably plague bulk materials, and to a lesser but still non-trivial extend also thin films.

\section{Conclusions}

We have shown that $\mathrm{Nb}_{3} \mathrm{Sn}$ 's large susceptibility to strain originates from significant sub-lattice distortions in the $\mathrm{Nb}$ chains in the A15 lattice. These distortions cause various bands to shift away from the Fermi energy, leading to a substantial reduction of the electron DOS at the Fermi energy level that is for more than $90 \%$ due to sub-lattice distortions. The reduction of $N\left(E_{\mathrm{F}}\right)$ is the dominant cause (about $80 \%$ ) of the reduction of the superconducting properties that is observed in experiments, whereas changes in the phonon spectrum only account for about $20 \%$ of this reduction. The amount of sub-lattice distortion, and hence the sensitivity to strain, is significantly larger for strain in the [100] direction than for strain in the [110] direction. These findings suggest two methods to eventually reduce the strain sensitivity in $\mathrm{Nb}_{3} \mathrm{Sn}$ wires: (1) by imposing a [110] crystal orientation along the wire axis; and (2) by stabilizing the $\mathrm{Nb}$ chains in the A15 lattice (for example through suitable dopants such as aluminum). These conclusions highlight possible ways to mitigate the reduction of the current carrying capacity by strain, which should enable $\mathrm{Nb}_{3} \mathrm{Sn}$ superconducting magnets to reach higher magnetic fields, yield more compact magnet windings, and simplify react-and-wind magnet construction.

\section{Acknowledgments}

This work was primarily supported by the Director, Office of Science, Offices of Basic Energy Sciences and High Energy Physics, of the US Department of Energy under Contract No. DE-AC02-05-CH11231; FH was supported by the Nonequilibrium Magnetic Materials Program (MSMAG). The authors would like to thank the Cornell NanoScale Science and Technology Facility and Berkeley's National Energy Research Scientific Computer Center for making their facilities available for this work, Dr W Goldacker for the fabrication of the bulk niobium-tin samples that were used in this 
study, and Dr A Anders and Dr J L Slack for their help and the use of the thin film deposition chamber that was used to fabricate the thin film samples.

\section{ORCID iDs}

A Godeke (10) https://orcid.org/0000-0002-8924-9878

\section{References}

[1] Ball A et al 2014 Future Circular Collider study hadron collider parameters CERN Technical Document FCC1401101315-DSC CERN (fcc.web.cern.ch)

[2] National Research Council 2005 Opportunities in High Magnetic Field Science (Washington, DC: The National Academic Press)

[3] National Research Council 2013 High Magnetic Field Science and its Application in the United States: Current Status and Future Directions (Washington, DC: The National Academic Press)

[4] Lee P J and Strauss B P $2011 \mathrm{Nb}-\mathrm{Ti}$-from beginnings to perfection 100 Years of Superconductivity ed H Rogalla and P H Kes (Boca Raton, FL: CRC Press) ch 11.2 pp 643-60

[5] Godeke A 2006 Supercond. Sci. Technol. 19 R68-80

[6] Flükiger R, Uglietti D, Senatore C and Buta F 2008 Cryogenics 48 293-307

[7] Godeke A 2005 Performance boundaries in $\mathrm{Nb}_{3} \mathrm{Sn}$ superconductors PhD Thesis University of Twente

[8] Senatore C, Abächerli V, Cantoni M and Flükiger R 2007 Supercond. Sci. Technol. 20 S217-22

[9] Godeke A, Jewell M C, Fischer C M, Squitieri A A, Lee P J and Larbalestier D C 2005 J. Appl. Phys. 97 093909

[10] Lee P J and Larbalestier D C 2008 Cryogenics 48 283-92

[11] Mentink M G T, Dhalle M M J, Dietderich D R, Godeke A, Hellman F and Ten Kate H H J 2017 Supercond. Sci. Technol. 30025006

[12] Xu X, Sumption M, Peng X and Collings E W 2014 Appl. Phys. Lett. 104082602

[13] Ekin J W, Cheggour N, Goodrich L and Splett J 2017 Supercond. Sci. Technol. 30033005

[14] Takeuchi T, Iijima Y, Inoue K, Wada H, ten Haken B, ten Kate H H J, Fukuda K, Iwaki G, Sakai S and Moriai H 1997 Appl. Phys. Lett. 71 122-4

[15] Ekin J W 1981 IEEE Trans. Magn. 17 658-61

[16] Bardeen J, Cooper L N and Schrieffer J R 1957 Phys. Rev. 108 $1175-204$

[17] Éliashberg G M 1960 Sov. Phys._JETP 11 696-702 Éliashberg G M 1960 J. Exp. Theor. Phys. 38 966-76

[18] McMillan W L 1968 Phys. Rev. 167 331-44

[19] Hopfield J J 1969 Phys. Rev. 186 443-51

[20] Kresin V Z 1987 Phys. Lett. A 122 434-8

[21] Ginzburg V L and Landau L D 1950 Zh. Eksp. Teor. Fiz. 20 1064-82

[22] Abrikosov A A 1957 Sov. Phys.-JETP 5 1174-82 Abrikosov A A 1957 J. Exp. Theor. Phys. 32 1442-52
[23] Gor'kov L P 1959 Sov. Phys._JETP 36 1364-7

Gor'kov L P 1959 J. Exp. Theor. Phys. 36 1918-23

[24] Rieck C T, Scharnberg K and Schopohl N 1991 J. Low Temp. Phys. 84 381-464

[25] Giannozzi P et al 2009 J. Phys.: Condens. Matter 21395502

[26] Testardi L R and Mattheiss L F 1978 Phys. Rev. Lett. 41 $1612-5$

[27] Mattheiss L F and Testardi L R 1979 Phys. Rev. B 20 2196-200

[28] Markiewicz W D 2004 Cryogenics 44 767-82

[29] Rudman D A and Beasley M R 1984 Phys. Rev. B 30 2590-4

[30] Devantay H, Jorda J L, Decroux M, Muller J and Flükiger R 1981 J. Mater. Sci. 16 2145-53

[31] Lu X F, Taylor D M J and Hampshire D P 2008 Supercond. Sci. Technol. 21105016

[32] Godeke A, Chlachidze G, Dietderich D R, Ghosh A K, Marchevsky M, Mentink M G T and Sabbi G L 2013 Supercond. Sci. Technol. 26095015

[33] Mentink M G T, Dhalle M M J, Dietderich D R, Godeke A, Goldacker W, Hellman F and ten Kate H H J 2012 AIP Conf. Proc. 1435 225-32

[34] Mentink M G T, Dhalle M M J, Diederich D R, Godeke A, Goldacker W, Hellman F, ten Kate H H J, Sumption M D and Susner M A 2012 Phys. Proc. 36 491-6

[35] Mentink M G T et al 2011 IEEE Trans. Appl. Supercond. 21 2250-3

[36] Mentink M G T 2014 An experimental and computational study of strain sensitivity in superconducting $\mathrm{Nb}_{3} \mathrm{Sn} P h D$ Thesis Univ. of Twente, Enschede, The Netherlands

[37] ten Haken B, Godeke A and ten Kate H H J 1997 Adv. Cryo. Eng. 42B 1463-70

[38] Poirier M, Plamondon R and Cheeke J D N 1984 J. Appl. Phys. 55 3327-32

[39] Muzzi L et al 2012 Supercond. Sci. Technol. 25054006

[40] Scheuerlein C et al 2014 Supercond. Sci. Technol. 27044021

[41] Scheuerlein C, Arnau G, Alknes P, Jimenez N, Bordini B, Ballarino A, Di Michiel M, Thilly L, Besara T and Siegrist T 2014 Supercond. Sci. Technol. 27025013

[42] Weber W and Mattheiss L F 1982 Phys. Rev. B 25 2270-84

[43] Sadigh B and Ozoliņ̌ V 1998 Phys. Rev. B 57 2793-800

[44] Axe J D and Shirane G 1983 Phys. Rev. B 28 4829-30

[45] Testardi L R 1971 Phys. Rev. B 3 95-106

[46] Markiewicz W D and Toth J 2006 Cryogenics 46 468-76

[47] Oh S and Kim K 2006 J. Appl. Phys. 99033909

[48] De Marzi G, Morici L, Muzzi L, della Corte A and Buongiorno Nardelli M 2013 J. Phys.: Condens. Matter 25 135702

[49] Valentinis D F, Berthod C, Bordini B and Rossi L 2014 Supercond. Sci. Technol. 27025008

[50] Qiao L and Zheng X 2012 J. Appl. Phys. 112113909

[51] Qiao L, Yang L and Song J 2015 Cryogenics 69 58-64

[52] Zhang R, Gao P and Wang X 2017 Cryogenics 86 30-7

[53] Godeke A, den Ouden A, Nijhuis A and ten Kate H H J 2008 Cryogenics 48 308-16

[54] Bordini B, Bottura L, Mondonico G, Oberli L, Richter D, Seeber B, Senatore C, Takala E and Valentinis D 2012 IEEE Trans. Appl. Supercond. 226000304

[55] Lu X F and Hampshire D P 2010 Supercond. Sci. Technol. 23 025002

[56] Sandim M J R, Sandim H R Z, Zaefferer S, Raabe D, Awaji S and Watanabe K 2010 Scr. Mater. 62 59-62 